\title{
Combining a Career in Clinical Practice and Research: The Benefits at Junior Career Level
}

\author{
*Jenny Hiley ${ }^{\mathrm{a}}$. Jed Jerwood ${ }^{\mathrm{b}}$, Jonathan Pricec, Siân Thomas ${ }^{\mathrm{d}}$, and Joyce Kenkre ${ }^{\mathrm{e}}$ \\ ${ }^{a}$ University Hospitals Birmingham NHS Foundation Trust, United Kingdom; ${ }^{b}$ Birmingham and Solihull \\ Mental Health Foundation Trust, United Kingdom, ${ }^{C}$ Birmingham Community Healthcare NHS Foundation \\ Trust, United Kingdom; ${ }^{d}$ Bwrdd Iechyd Prifysgol Aneurin Bevan/Aneurin Bevan University Health Board, \\ United Kingdom; ${ }^{e}$ University of South Wales, United Kingdom
}

\begin{abstract}
Clinical academic careers programmes have developed in England and Wales to enable clinical staff outside of medical and dentistry - namely Nurses, Midwives, Allied Health Professionals, Pharmacists and Healthcare Scientists (NMAHPPS) to develop their academic and research skills alongside clinical practice. These schemes have complemented preexisting national clinical academic careers pathways for Nurses, Midwives and Allied Health Professions (NMAHPs). Multi-professional case studies from the West Midlands and Wales are used to illustrate the benefits of clinical academic careers for individuals at junior career (pre-doctoral) level. The following case studies will be included: Jed Jerwood, an art psychotherapist, who is aspiring to be an evidence-based practitioner; Jonathan Price, a physiotherapist, who is developing support networks and navigating the system; and Siân Thomas, a nurse, who is developing opportunities to influence local and national practice. The benefits of clinical academic careers and the support from the English and Welsh programmes can be demonstrated in individuals at junior career level. A range of benefits are described including the emergence of autonomous evidence-based practitioners, developing their networks and collaborations, along with a plan for the future.
\end{abstract}

Keywords: evidence-based practitioner; healthcare provider organisation; internship; junior clinical academic; knowledge economy skills scholarships; research;

\section{Introduction}

This article uses personal case studies of three clinicians who have completed clinical academic career programmes developed in the West Midlands and Wales to illustrate the benefits of clinical academic roles for individuals at junior career level. In this case, junior career level describes clinicians who are experienced in clinical practice, but who have limited or no experience of undertaking their own research. Drawing upon their individual experiences, this article highlights the value of undertaking a structured training programme, guided by supervisors and mentors in developing the necessary skills and knowledge

\footnotetext{
*Corresponding Author: Jenny Hiley, Institute of Translational Medicine, Mindlesohn Way, Edgbaston, Birmingham B15 2TH United Kingdom $\quad$ Email: jenny.hiley@uhb.nhs.uk
}

Journal URL: https://publications.coventry.ac.uk/index.php/pblh

Hiley. J., Jerwood, J., Price, J., Thomas, S., \& Kenkre, J. (2019). Combining a career in clinical practice and research: The benefits at junior career level. International Journal of Practice-based Learning in Health and Social Care, 7(2), 36-45. DOI 10.18552/ijpblhsc.v7i2.638

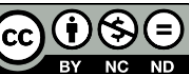
Access article is distributed under the terms of the Creative Commons Attribution Attribution-Non-Commercial No Derivatives 4.0 International License (https://creativecommons.org/licenses/by-nc-nd/4.0/ ), which permits unrestricted non-commercial use, distribution, and reproduction in any medium, provided the original work is properly cited and is unaltered. 
to embark on a clinical academic career in the National Health Service (NHS). It also discusses some of the challenges faced by clinicians at the early stages of their career.

\section{Background}

The editorial by Carrick-Sen and Moore (2019), elsewhere in this special issue of the International Journal of Practice-based Learning in Health and Social Care, describes the UK national pathways that have been created to support and develop clinical academic healthcare professionals outside of medicine and dentistry; namely: Nurses, Midwives and Allied Health Professions (NMAHPs) in England since 2008, and in Wales since 2005. In this article, we consider the expanded pathways established in 2014, namely, Nurses, Midwives, Allied Health Professionals, Pharmacists and Healthcare Scientists (NMAHPPS).

\section{Developing Clinical Academic Careers: The English Model}

In England, to support entry onto the Health Education England (HEE)/National Institute for Health Research (NIHR) Integrated Clinical Academic (ICA) Programme (HEE/NIHR, 2019a), regional HEEfunded clinical academic careers bridging schemes were introduced in 2014. Three programmes are currently delivered in the West Midlands providing individuals with the skills and knowledge needed to develop a clinical academic career and successfully apply for further formal training (Birmingham Health Partners, 2019).

- Internship Programme - a pre-Masters programme offering an introduction to clinical academic careers and preparation for Masters level study.

- Pre-Doctoral Bridging Programme- bridging the gap between Masters and doctoral level study.

- Post-Doctoral Bridging Programme - bridging the gap between doctoral study and application for clinical lectureship or equivalent.

Figure 1 illustrates the West Midlands programmes in relation to the ICA pathway.

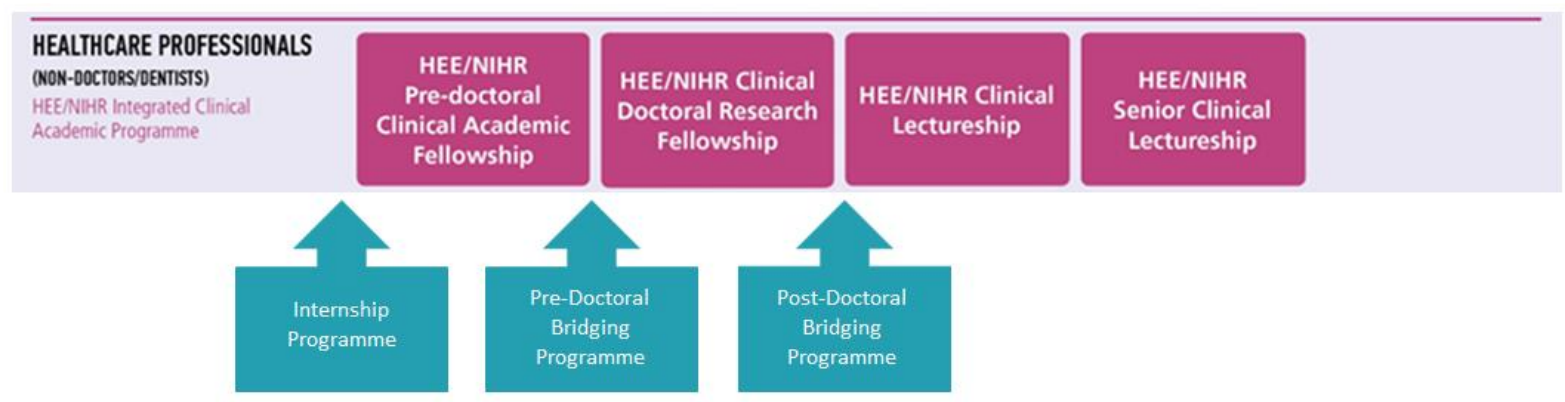

Figure 1 - The West Midlands clinical academic careers programmes in relation to the HEE/NIHR Integrated Clinical Academic Programme for non-medical healthcare professions (Figure adapted with the kind permission of NIHR)

The West Midlands clinical academic careers programmes are delivered by the Integrated Clinical Academic Office (ICAO) team, which is part of Birmingham Health Partners (BHP), a collaboration between University Hospitals Birmingham NHS Foundation Trust, Birmingham Women's and Children's NHS Foundation Trust, and the University of Birmingham (UoB). The pre-Masters Internship Programme (formerly Clinical Academic Internship Programme [CAIP]) was piloted in 2014, offering a taster of clinical academic and research for NMAHPPS, the first stage of the HEE/NIHR ICA programme. A further five programme cohorts have completed to date $(n=97)$. The Internship Programme equips individuals with the knowledge and skills to undertake their own research, critique current evidence and apply findings to their practice to improve patient care. It assists individuals with developing a research question and their clinical academic career profile. The 2019 intake is delivered in 
48 days over a period of 12 months and includes the provision of a taught programme enabling interns to develop skills in the design and delivery of clinical research, access to observational and practical placements within a research or academic environment and one-to-one support from a clinical academic supervisor. The expected programme outcomes include:

- A refined and feasible research question as part of a preliminary research proposal in which potential methods are considered;

- A literature review supporting the research question formatted for submission to a nominated journal;

- Portfolio containing evidence of learning and experiences of the novice researcher while on the programme to include self-assessment of ongoing learning needs and future plans;

- Completed Masters in Clinical Health Research module;

- Application to the next stage of the clinical academic pathway;

- Dissemination of programme work to the intern's employing organisation.

This programme supports application to the next stage of the HEE/NIHR ICA pathway, the Pre-doctorate Clinical Academic Fellowship (PCAF) or other equivalent training such as a Masters in Clinical Health Research. To gain postgraduate credits and an insight into this level of study, Internship Programme participants also have the opportunity to complete a Masters-level module at the UoB.

A review of the Internship Programme identified that individuals also needed support to apply for doctorate-level study, and the HEE-funded Masters to Doctorate Bridging Programme (now Pre-Doctoral Bridging Programme) was developed in 2015. This programme provides greater emphasis on the development of leadership skills and building of a research proposal and clinical academic career profile in preparation for doctoral level application. To date, four Pre-Doctoral Bridging Programme cohorts have completed $(n=30)$.

To support the gap between doctoral-level study and applying for a clinical lectureship or equivalent funding, a Post-Doctoral Bridging Programme was developed in 2018. This supported two individuals in the design of a programme of research, and also to develop a clinical and academic mentorship team to support applications for post-doctorate training. The second cohort $(n=3)$ commenced in October 2019 and will provide 48 days over a 12-month period. It will also offer individuals a funding grant to support their development, which will include salary support, training, external visits and publication costs.

Individuals accessing the West Midlands clinical academic careers programmes benefit from the support of clinical academic supervisors, selected from a pool of over a hundred experienced post-doctoral academics, working in a range of clinical disciplines across the region and beyond. These supervisors are given a one-off honorarium.

\section{Clinical academic careers: the Welsh model}

The Research Capacity Building Collaboration (RCBC) Wales was created in 2005 and was originally jointly funded by the Health Foundation and the Welsh Office for Research and Development (WORD) (RCBC Wales, 2019). Following the publication of the 'Finch Report' (Finch Group, 2012), there was an increase in funding from WORD and the Higher Education Funding Council for Wales (HEFCW). This was to increase the capacity and capability of nurses and midwives to conduct research that would have an impact on clinical practice. It was decided within the Welsh Government to include allied health professionals in this initiative to give them the opportunity to develop their evidence base for practice as well. It is now funded by Health and Care Research Wales (Welsh Government) with additional funds provided by Tenovus Cancer Care and now covers 20 healthcare professional groups including pharmacists. The types of awards available are:

- $\quad$ First into Research (FiR)

- $\quad \mathrm{PhD}$

- Post-Doctoral

- Early career research

- Senior Career Research Fellow. 
So far, 89 healthcare professionals have been awarded RCBC fellowships.

The key factor in creating a collegiate environment across disciplines and the shared infrastructure and support provided between six university nursing and allied health departments has been the Community of Scholars. Attendance is mandatory, as agreed when applying for a fellowship. The Community of Scholars have bi-monthly meetings including one residential each year. Issues covered include a range of research methodologies, skills, leadership, impact, political awareness and media training.

However, running alongside this scheme is the Knowledge Economy Skills Scholarships (KESS), funded by the European Social Fund (ESF) with matched funding from industry (the industry can be a Health Board) (Bangor University, 2019). From 2009 to 2023 the grant funding for PhDs and Masters in Research (MRes) is $£ 87.5$ million of which $£ 58.5$ million is from the ESF. Scholars have their fees covered, receive a bursary and research expenses, and are expected to present at conferences nationally and internationally within their budget. The total budget is to cover the cost of $708 \mathrm{PhDs}$ and $600 \mathrm{MRes}$, which could be considered as junior career level awards. This has been possible as most of Wales is within the European Convergence Area. Fifty percent of the scholarships have been awarded to life sciences and healthcare professionals.

Every KESS 2 scholar attends a residential KESS Grad School to work on business-related training and development activities. Through the European Universities Association (EUA) the European Industrial Doctoral School (EIDS) has been established, which means the scholars can work with partner universities across Europe and offer transnational opportunities to the scholars and their company partners.

The objectives of the KESS awards are:

- To increase the research capacity of small to medium enterprises (SMEs) by linking with a PhD / Research Masters project;

- To encourage SMEs to undertake research and to recruit researchers;

- To prepare and train individuals to contribute to research as professionals;

- To support the development of key technologies in the Convergence Area of Wales;

- To promote higher-level skills development.

This is in response to the Welsh Governments grand challenge areas:

- $\quad$ Life Sciences \& Health;

- Advance Engineering \& Materials;

- Low Carbon, Energy \& Environment;

- Information and Communications Technology (ICT) \& the Digital Economy.

As the RCBC and KESS awards both go through a competitive process, the scholars are all eligible to apply to go on the research portfolio in Wales. This means that they qualify for support from the research infrastructure and the research participants can be counted in each of the health boards' recruitment figures.

\section{Method}

Multi-professional case studies (an art psychotherapist, a physiotherapist and a nurse) from the West Midlands and Wales are used to illustrate the benefits of clinical academic careers for individuals at earlycareer level. These are set out below. 


\section{Case study 1: Becoming an evidence-based practitioner (Jed Jerwood)}

This case study illustrates how, following attendance on the West Midlands Internship Programme, Jed Jerwood developed as a confident evidence-based practitioner.

Jed works as a Senior Art Psychotherapist at Birmingham and Solihull Mental Health Foundation Trust and as a clinical academic researcher on the Improving End of Life Care in Mental Health Project.

Jed's research centres around understanding and improving palliative and end of life care for people with mental illness and co-existing terminal conditions. He undertook the Internship Programme in 2015/16.

Jed applied to the internship programme as he was interested in combining a career in research and academia with his clinical role. He also had an aspiration to undertake a $\mathrm{PhD}$ and believed the programme would give him the skills and knowledge that he would need to apply in the future. He had started to develop a research idea but wanted to increase his knowledge of how to undertake clinical research in a healthcare setting. He initially applied to undertake a Masters in Clinical Health Research but was unsuccessful in gaining a place. Once he embarked the internship programme he realised why this had been the case, feeling that he lacked the understanding of the skills needed to critique the evidence, refine his research question and present his research ideas in an appropriate way.

As a result of attending the internship programme Jed developed the skills needed to become a confident evidence-based practitioner in the NHS and is now able to interrogate current evidence to pilot novel interventions and undertake service redesign. He set up a research group at the hospice where he worked at the time and also supports colleagues in the development of their research ideas. Furthermore he has written a palliative and end of life care strategy at his NHS Trust and set up a working group, chaired by the Director of Nursing, to implement the strategy across the Trust.

Jed also was successful in gaining an HEE/NIHR-funded place on the Masters in Clinical Health Research at Coventry University the following year (this was the second stage of the HEE/NIHR ICA programme until the Pre-doctoral Clinical Academic Fellowship was introduced in 2018). Following this he was awarded a doctoral place and scholarship with Coventry University to develop his clinical academic research further through doctoral study. He completed his $\mathrm{PhD}$ this summer. Jed has been an invited speaker at several national events and delivered webinars for HEE and NHS England as part of a series on marginalised groups in palliative and end of life care. He has attended the European Association of Palliative Care Congress in Berlin in May 2019 to present his research findings.

He has recently returned to clinical practice and received promotion to a more senior role, and has been successful in gaining post-doctoral funding from his NHS Trust and Birmingham Health Partners to develop the next stage of his research proposal for a post-doctoral full award.

\section{Case study 2: Developing support networks and navigating the system (Jon Price)}

This case study outlines how Jon Price developed valuable supervisory and mentor relationships as a result of attending the Internship Programme.

Jon works as an MSK (Musculoskeletal) Physiotherapy Research Lead and Advanced Physiotherapist at Birmingham Community Healthcare NHS Foundation Trust (BCHC).

Jon's research area is neck pain rehabilitation. As part of his Research Lead role Jon acts as Site Coordinator on two NIHR-funded National Portfolio randomised controlled clinical trials. He undertook the Internship Programme in 2017/18.

Jon's interest in research stemmed from his undergraduate dissertation while studying Physiotherapy at the University of Birmingham for which he was awarded the 'Dissertation of the Year 2010' Award. During his work in private practice he re-established his links with the university to work as research coordinator on a Royal Thai Government-funded study investigating whiplash interventions, before joining BCHC and later taking up the position of MSK Physiotherapy Research Lead. Jon undertook the 
internship programme to provide him with formal training in research methods and to enable him to develop as an independent research practitioner, whilst maintaining his NHS contract and salary.

Completing the internship programme has given Jon the opportunity to develop his support networks as he embarks on his clinical academic career. As part of the programme he was matched to a suitable clinical academic supervisor with a similar research area of interest and professional background. This supervisory relationship has been essential in helping Jon to focus his research ideas and undertake a systematic review of the evidence.

The programme also enabled participants to learn from current clinical academic role models. Although Jon has had the support of his employer and their strategic commitment to develop clinical academic posts for NMAHPPS, he recognised the need for mentorship from those with more experience of balancing academic and clinical workloads and navigating the system. He invited one of the programme speakers - a current NIHR Clinical Doctoral Research Fellowship trainee (CDRF) - to act as his mentor.

These supervisory and mentorship relationships have continued after the programme concluded, both of which assisted Jon to apply for the HEE/NIHR Pre-doctoral Clinical Academic Fellowship (PCAF). Jon was successful in his application and commenced this fellowship in September 2018, giving him the time to work on his research proposal and develop his application for the CDRF.

\section{Case study 3: Opportunities for influencing local and national practice (Siân Thomas)}

This case study illustrates how the First into Research Fellowship has enabled Siân to influence both local and national practice.

Siân is a Consultant Nurse in Child Health in the Aneurin Bevan University Health Board, Wales.

Being part of the Research Capacity Building Collaboration First into Research fellowship has been very rewarding and motivating for Siân. It has enabled her to develop her research skills, knowledge and confidence in this area and to continue the pathway towards Doctoral study.

Having identified the need for the clinical research it was found to be really beneficial to develop the evidence base in this area to influence innovation in clinical practice, both locally and nationally. The findings from the study have enabled Siân and her team to develop guidance to reduce inappropriate variation, promote best practice and optimise patient safety in her clinical area.

There were many positive elements of the First into Research (FiR) fellowship, namely the peer support network offered through the Community of Scholars $(\mathrm{CoS})$, which enabled discussion and debate with like-minded clinical researchers as well as being a source of professional development. Additionally, the link with a higher education institution and access to one-to-one mentorship from an academic supervisor were very helpful. Being part of this scheme widened Siân's networks and enabled/influenced research conversations, giving her the confidence and motivation to look for further opportunities and develop national and international links. An example of this is having successfully secured an International Travel scholarship to further understand the area of research as well as being invited to join a UK Blended Diet Research Collaborative.

As the scheme is only funded for a year, it was helpful that the research proposal and ethics process were well developed from the outset; however, the timeframe for completion of study was quite challenging.

As a result of research dissemination Siân has been invited to deliver oral presentations at national conferences and meetings, and she plans to publish in medical, nursing and dietetic journals which will influence a change in clinical practice. 


\section{Discussion}

This article has aimed to illustrate the benefits of clinical academic roles for individuals at junior research career level. Personal case studies by three clinicians who had completed clinical academic career programmes developed in the West Midlands and in Wales have been used to demonstrate the different aspects of the training fellowships and the impact it has had in developing their research knowledge, skills and expertise.

\section{Becoming an evidence-based practitioner}

The clinical academic careers programmes are attended by a wide range of highly experienced and skilled professionals (Hiley et al., 2018; RCBC Wales, 2019). Programme participants bring a wealth of clinical expertise, ideas from their clinical experience, and a desire to improve care delivery. However many often do not fully understand the research process, i.e. to take an idea, to define the question and then develop an appropriate design to be able to interrogate the problem in order to find an evidence-based solution. The development of critical appraisal skills is an important outcome of the West Midlands careers programmes. An evaluation of the West Midlands programmes reported that the majority of respondents had more confidence in questioning their clinical practice, and to search and appraise current literature, as a result of attending the programme (Hiley et al., 2018).

Jed described how the programme had increased his confidence in his clinical practice and enhanced his understanding of the value of research on patient care:

I think I am a more confident practitioner as a result and have a greater understanding of how clinical academic research supports changes in clinical practice with direct patient benefit (Hiley et al., 2018; Health Education England, 2019)

The literature outlines the positive benefits for healthcare as a result of clinical staff involvement in research (Boaz et al., 2015). For Jed, this renewed level of confidence as a research-literate practitioner gave him the impetus needed to influence changes in practice in his organisation, to promote a positive research culture and to support his colleagues to develop as well. This also resulted in him building his clinical academic career profile and completing valuable research outputs including oral presentations of his work. He was also more equipped to apply for postgraduate training and doctoral opportunities.

Another aspect of training is the importance of the dissemination of information, not necessarily just through publication of research in journals, but in constructive engagement with the media and policy makers. An important aspect of the training in Wales addresses such issues, to ensure you get your point over to the media, including practice and feedback. As Wales has a devolved government, RCBC fellows have the opportunity to spend time with policy makers in the Welsh Government and ultimately have the opportunity to present their issue forward to Welsh Assembly members. This gives the fellows the expertise of focusing down on the key points that they want to put across, based on factual information.

\section{Listening to the needs of the patients and public}

It is now part of the culture of research to have patient and public engagement but often it is seen as a token gesture. However, if somebody is living with a condition and trying to cope with it on a daily basis they themselves possess a wealth of knowledge and ideas about their current and future needs. Until quite recently children were excluded from research but with the changing technical opportunities, they are often ahead of us in the potential use of technology to enhance their care and wellbeing.

The importance of listening to the needs of children and their families cannot be overemphasised. Parents of children with long-term health conditions are increasingly considered as experts in their child's care, so it is important to take their views meaningfully into account as it enriches decision-making processes when planning and developing services. Enabling families to share their perspective can improve future care provision by influencing service change.

The area of practice that was researched by Siân was triggered by the needs and wishes of a parent; professionals were in fact uncertain about the practice due to lack of clinical evidence. The development 
of clinical guidelines and risk-assessed approach provided the platform for the research study to develop the evidence base and inform national practice.

\section{Developing support networks and navigating the system}

Junior clinical academics can require assistance to refine their research ideas and utilise research methodologies. The clinical academic programmes provided the necessary support through the use of clinical academic supervisors. Not only did these supervisors equip their supervisees with the research skills and knowledge to begin to develop their independence as research practitioners, they also provided assistance with navigating the research system, in terms of research governance, ethics, costings and working within the NHS. This supervisory relationship was highly regarded by previous programme participants in an evaluation of the West Midlands programmes (Hiley et al., 2018).

Despite the introduction of a nationally funded clinical academic career pathway for NMAHPPS, there are few career structures for trainees returning to NHS practice (Bramley et al., 2018; Carrick-Sen et al., 2016). Jon's employer was wholly supportive of him undertaking the Internship Programme, developing his clinical academic profile and clinical academic role within the organisation. The benefits of cohort learning on the programme meant that Jon could share his experiences of engaging with this clinical manager and his organisation's Research and Innovation team to devise a plan for his development enabling factors not experienced by all on the programme. Examples shared included the value of succession planning when preparing for his release from clinical practice to undertake his PCAF training and the need to align his research ideas with organisational and team priorities.

Teaching sessions were delivered by a range of experienced NMAHPPS clinical academics to assist programme participants to gain an insight into the varied career paths and how to overcome the challenges faced when embarking on these. Teaching was structured using the Vitae Researcher Development Framework (Vitae, 2011). Research sessions included: how to develop a research question and proposal; quantitative and qualitative research methods; critical appraisal and literature reviews; applying research methodologies to a healthcare setting; patient and public involvement and engagement; and ethics and research governance. Individuals were also encouraged to develop their clinical academic profile with an introduction to the clinical academic career pathways, funding and finance, presenting with impact, writing winning posters and abstracts.

Although Jon had the support of his employer, he felt he required more personal support both with this and to build a mentorship relationship:

I was inspired by his career path and witnessing his commitment and desire to develop as a clinical academic... he is an ideal role model and clinical academic mentor as I will emulate his route.

The importance of role models to support junior clinical academics has been reported in the literature (Bramley et al., 2018; Fullam et al., 2018) and cannot be underestimated. The introduction of a mentorship scheme to support the HEE/NIHR ICA programme (HEE/NIHR, 2019b) reinforces the value that a mentor relationship can bring.

Jon also reported that in completing the clinical academic programme he gained valuable access to networks of clinical academics in the region, including supervisors, taught-programme speakers, programme alumni, and the programme delivery team. Through this and as a programme alumnus, Jon has since become a founding member of the newly formed West Midlands Alliance of Research Networks, with the BHP ICAO team and the NIHR Clinical Research Network West Midlands; two of its aims being to support the development of junior NMAHPPS researchers, and to enable multidisciplinary research collaborations across the West Midlands.

In the development of her research even at these early stages, Siân has encountered the need to address protection of intellectual property. It has to be recognised now, with the generation of new ideas and new ways of working, that we need to inform and engage with agencies to support our practitioners in developing and engaging with industrial partners to develop an idea, through research into a product for market. 


\section{Intellectual property}

Each employing organisation should adopt guidance for the protection of intellectual property so that it is not lost to the organisation or the person who has developed the original idea. This should include process and costs, as well as distribution of financial benefits for the organisation and the person who has developed the original idea (which may be more than one person working as a team). Protecting intellectual property should not be seen as a barrier to the development of innovative ideas and products. However, when engaging with companies it may be wise to have non-disclosure agreements in place at an early stage of development.

\section{Brexit}

The withdrawal from the European Union (EU) will have an impact on a wide range of issues including recognition of professional accreditation across European countries, employment, access to medications, authorisation of drugs and equipment, and the ability to collaborate in research (Fahy et al., 2019). The most recently proposed Withdrawal Agreement will secure continuity for current European Investment Bank-funded projects, but no new projects after March 2019, because future funding will be reserved for member states. This will have a huge impact on the economy especially in Wales, which received extensive EU 'Convergence' funding to improve the economy and infrastructure. It will mean that Britain will not be represented on important European expert groups except as an invited expert. Consequently, we need to reposition our research and access non-UK research funding and create new global opportunities and collaborations.

\section{Innovation}

The World Health Organisation (WHO) Health Innovation group defines innovation (WHO, 2019):

Health innovation is to develop and deliver new or improved health policies, systems, products and technologies, and services and delivery methods that improve people's health.

We need our training programmes to ensure that our junior clinical researchers understand the infrastructure and support available to them within their own employing organisation, regionally, nationally and internationally to increase the impact of their research, especially if it establishes the benefit of a new product.

\section{Conclusion}

The case studies illustrate that individuals may chart their own personal clinical academic career journeys. The value of attending structured programmes enables individuals at junior career level to develop the essential skills required to become autonomous evidence-based practitioners, whilst developing collaborations and supportive networks, both to strengthen their work and to assist with planning their future direction. However, in our training we need to ensure that we embrace the dissemination of information through the media, in clinical practice, and to policy makers, and, moreover, that we support the development of our international networks and collaborations for the future of UK research.

\section{ORCID}

Jed Jerwood: $\quad$ https://orcid.org/0000-0003-1135-3111

Jonathan Price https://orcid.org/0000-0002-5505-1564

Joyce Kenkre: $\quad \underline{\text { https://orcid.org/0000-0002-2067-9001 }}$

\section{References}

Bangor University. (2019). Doctoral School: Knowledge Economy Skills Scholarships (KESS) 2. https://www.bangor.ac.uk/doctoral-school/kess/index.php.en 
Birmingham Health Partners (BHP). (2019). Clinical Academic Careers Programmes. https://www.birminghamhealthpartners.co.uk/education/clinical-academic-careers-programmes/

Boaz, A., Hanney, S., Jones, T., \& Soper, B. (2015). Does the engagement of clinicians and organisations in research improve healthcare performance: A three-stage review. BMJ Open, 5(12), e009415. https://doi.org/10.1136/bmjopen-2015-009415

Bramley, L., Manning, J. C., \& Cooper, J. (2018). Engaging and developing frontline clinical nurses to drive care excellence: Evaluating the Chief Nurse Excellence in Care Junior Fellowship initiative. Journal of Research in Nursing, 23(8), 678-689. https://doi.org/10.1177/1744987118808843

Carrick-Sen, D. M., \& Moore, A. P. (2019). Editorial: Improving care and outcome through NMAHP research-focused clinical academic roles - An international perspective. International Journal of Practice-based Learning in Health and Social Care, 7(2), ii-vi. DOI 10.18552/ijpblhsc.v7i2.648

Carrick-Sen, D., Richardson, A., Moore, A., \& Dolan, S. (2016). Transforming healthcare through clinical academic roles in nursing, midwifery and allied health professions: A practical resource for healthcare provider organisations. https://councilofdeans.org.uk/wpcontent/uploads/2019/02/AUKUH-Transforming-Healthcare.pdf

Fahy, N., Hervey, T., Greer, S., Jarman, H., Stuckler, D., Galsworthy, M. \& McKee, M. (2019). How will Brexit affect health services in the UK? An updated evaluation. The Lancet, 393: 949-958. https://doi.org/10.1016/S0140-6736(19)30425-8

Finch Group. (2012). Accessibility, sustainability, excellence: how to expand access to research publications. Report of the working group on expanding access to published research findings. https://www.acu.ac.uk/research-information-network/finch-report-final

Fullam, J., Cusack, E., \& Nugent, L. E. (2018). Research excellence across clinical healthcare: A novel research capacity building programme for nurses and midwives in a large Irish region. Journal of Research in Nursing, 23(8), 692-706. https://doi.org/10.1177/1744987118806543

Health Education England. (2019). Research Internships. https://www.hee.nhs.uk/our-work/clinicalacademic-careers/research-internships

Health Education England (HEE)/National Institute for Health Research (NIHR). (2019a). HEE/NIHR Integrated Clinical Academic Programme. https://www.nihr.ac.uk/our-researchcommunity/NIHR-academy/nihr-training-programmes/nihr-hee-ica-programme/

Health Education England (HEE)/National Institute for Health Research (NIHR). (2019b). ICA Mentorship Scheme. https://www.nihr.ac.uk/our-research-community/NIHR-academy/nihrtraining-programmes/nihr-hee-ica-programme/nihr-hee-ica-mentorship

Hiley, J., Begg, C., Banks, L., Harper, L., Swift, A., \& Topping, A. (2018). West Midlands Clinical Academic Careers Programmes for Nurses, Midwives, Allied Health Professions, Pharmacists and Healthcare Scientists (NMAHPPS); Evaluation report. Birmingham Health Partners.

RCBC Wales. (2019). RCBC Wales $\underline{\text { www.rcbcwales.org.uk }}$

Vitae. (2011). About the Vitae Researcher Development Framework. https://www.vitae.ac.uk/researchersprofessional-development/about-the-vitae-researcher-development-framework/about-the-vitaeresearcher-development-framework

World Health Organisation (WHO). (2019). WHO Innovation Group. https://www.who.int/lifecourse/about/who-health-innovation-group/en/ 